\title{
Physical connections: prokaryotes parasitizing their kin
}

\author{
Purificación López-García* and David Moreira
}

Ecologie Systématique Evolution, CNRS, Université Paris-Saclay, AgroParisTech, 91400 Orsay, France

*For correspondence: puri.lopez@u-psud.fr

Running head: Prokaryotes parasitizing their kin 


\section{The cooperation - competition continuum}

Microorganisms rarely live in isolated populations but thrive in complex communities where intricate interactions take place. These may have different effects on interacting community members, varying from synergistic to antagonistic along a cooperation - competition continuum. Between more classically studied antagonistic interactions, such as competition for a particular resource, and mutually beneficial interactions, such as the collective bacterial degradation of particular compounds extracellularly, there is room for a wide variety of interaction types according to their effects on organismal fitness. For instance, predation and parasitism benefit some community members to the expense of others. Commensal interactions, such as those that can be sometimes established along a trophic chain (e.g. the bacterial consumption of exopolymers secreted by photosynthesizing cyanobacteria), are profitable for some community members without noticeable effect on others. Together with abiotic factors, species interactions determine community structure (Little et al., 2008; Nadell et al., 2016) and impact evolution (Celiker and Gore, 2013; Mitri and Foster, 2013). The positive or negative influence and the strength of direct and indirect interactions in communities may vary with space and time, although how time regulates interactions is not yet well understood (Gorter et al., 2020). Ultimately, the selection of traits and, hence, the nature of interactions depend on the environment, which may favor or disfavor relations depending on their effects on fitness (Figure 1). Among the myriad of possible facultative interactions that can occur in a given ecosystem, some are

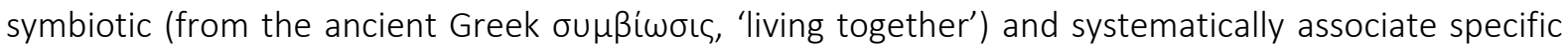
partners that interrelate more closely. Stable symbioses may rapidly become obligatory. This is facilitated by the physical association of partners along with gene loss and/or gene transfer from one partner to the other, which result in genome streamlining (McCutcheon and Moran, 2012; Moran and Bennett, 2014; Husnik and Keeling, 2019). Interactions among symbiotic partners can also occur along a cooperation - competition gradient, with mutualism being beneficial for both partners and parasitism, on the other end, having negative fitness effect on the host. Likewise, the nature of symbiotic interactions is likely to be influenced by the environment (Figure 1). Thus, some parasitic interactions can become mutualistic under certain circumstances. Even viruses, like plasmids and other selfish elements, which are intrinsic molecular parasites, can evolve mutualistic interactions, notably via temperate forms (Roossinck, 2011; Obeng et al., 2016; Roossinck and Bazán, 2017).

Studying interactions in the microbial world is not an easy task. Apart from the well-studied symbioses that led to the origin of mitochondria and plastids from, respectively, alphaproteobacteria and cyanobacteria (Schwartz and Dayhoff, 1978; Dyall et al., 2004), the best-known studied symbioses involve prokaryotes or microbial eukaryotes (e.g. mycorrhiza) interacting with animal or plant hosts (Dubilier et al., 2008; Moya et al., 2008; Delaux et al., 2013; Gourion et al., 2015). Among these, the 
alphaproteobacterial genus Wolbachia illustrates particularly well the mutualism-parasitism continuum. Members of this genus are transmitted vertically through host eggs and display a multitude of interactions with their invertebrate hosts. Mostly parasitic, they influence the host biology in various ways, often manipulating reproductive traits, including feminization, parthenogenesis, male killing, sperm-egg incompatibility (Werren et al., 2008) and host sex determination (Cordaux et al., 2011; Leclercq et al., 2016); but they can also be nutritional mutualists growing in dedicated host cells (bacteriocytes) (Hosokawa et al., 2010). Less well-known, often cryptic and focus of increasing research interest, symbioses of prokaryotes and protists are also widespread, especially in suboxic environments (Nowack and Melkonian, 2010; Keeling, 2013; Lopez-Garcia et al., 2017; Husnik and Keeling, 2019). In most cases, prokaryotes are protist endosymbionts, although several instances of epibiotic symbioses are known, e.g. involved in nutrient transfer (Thompson et al., 2012) or magnetotaxis (Monteil et al., 2019). However, until recently, known cases of prokaryote-prokaryote symbioses were relatively sparse and little understood, with the notable exception of metabolic symbioses (syntrophies) established between certain metabolic guilds (e.g. methanogenic archaea and fermentative bacteria, methanotrophic archaea and sulfate-reducing bacteria) (Fenchel and Finlay, 1995; Lopez-Garcia et al., 2017).

Syntrophies involving bacteria and/or archaea are widespread in sediments and other oxygendepleted settings where redox exchange fuels the degradation of complex organic matter and exergonic reactions are possible by the involvement of redox scavengers acting as metabolic sinks. Syntrophy typically involves the transfer of hydrogen or formate, but also of organic, sulfur- and nitrogen-compounds and even, directly, electrons (Sieber et al., 2012; Morris et al., 2013; Lovley, 2017). Most syntrophies are thought to be mutualistic but electron exchange might also involve competitive or parasitic interactions (Moscoviz et al., 2020). Although syntrophic interactions between prokaryotes are thought to be prevalent in anoxic environments, the identity of the partners and exchanged metabolites largely remain to be deciphered. This is partly due to the fact that these environments are complex and diverse, often displaying high cell-density, but also to the fact that most syntrophic interactions are facultative and/or cells are not physically linked. It is therefore very difficult to demonstrate specific metabolic symbioses unless in situ experiments, such as isotope-labeling coupled to high-resolution ion mass spectroscopy (e.g. nanoSIMS), can be applied to natural samples and/or cocultures can be obtained in the laboratory. Enriching and maintaining syntrophic consortia in culture is challenging. For instance, it took over a decade to isolate the first cultured Asgard archaeon in cooccurrence with its hydrogen-scavenging partners (either a methanogenic archaeon and/or a sulfatereducing deltaproteobacterium) (Imachi et al., 2020). Asgard archaea, famous for their close phylogenetic relatedness with eukaryotes, were previously known only from metagenome-assembled 
genomes (Spang et al., 2015; Zaremba-Niedzwiedzka et al., 2017). Their isolation and actual implication in syntrophic interactions lend serious credit to models for the origin of the eukaryotic cell implying that eukaryotes evolved from metabolic (endo)symbioses involving archaeal and bacterial partners (López-García and Moreira, 2020a). Yet, endosymbiosis of prokaryotes in prokaryotes has been unambiguously documented only for the nested occurrence of gammaproteobacteria within betaproteobacteria in mealybugs (von Dohlen et al., 2001) and that of alphaproteobacteria within mitochondria (derived alphaproteobacteria) in ticks (Sassera et al., 2006).

\section{Expanding prokaryote - prokaryote symbioses: CPR and DPANN phyla}

In recent years, it has nonetheless become increasingly clear that obligatory prokaryote-prokaryote symbioses implying a close physical connection are much more widespread than ever thought. Examples of tight cellular consortia, such as 'Chlorochromatium aggregatum', formed by a central motile heterotrophic betaproteobacterium and several peripheral anoxygenic photosynthesizing Chlorobi (Overmann and Van Gemerden, 2000), were previously known but rare. However, the discovery of extremely diverse lineages of likely symbiotic bacteria and archaea thanks to a combination of metabarcoding and metagenomic approaches with high-resolution microscopy is opening an unforeseen new window into the world of obligatory prokaryote-prokaryote interactions. In 2002, Huber and coworkers described Nanoarchaeum equitans, a nanosized archaeon ( $\sim 00 \mathrm{~nm}$ cell diameter) physically associated to the hyperthermophilic archaeon Ignicoccus hospitalis (Huber et al., 2002) (Figure 2). Its genome was very small (barely $0.5 \mathrm{Mbp}$ ) and lacked genes for lipid, cofactor, amino acid and nucleotide biosynthesis, which led to the conclusion that Nanoarchaeum was parasitic (Waters et al., 2003). Later studies, while confirming the obvious dependence of Nanoarchaeum on its specific host, did not necessarily show a negative impact on Ignicoccus. They rather suggested a peculiar symbiotic interaction enabling efficient inorganic carbon and nitrogen assimilation in the context of low energy yield sulfur-hydrogen respiration that deviated from strict mutualism, commensalism and parasitism definitions (Jahn et al., 2008; Podar et al., 2008). Subsequently, other nanosized archaea endowed with reduced genomes were described in association to other archaeal hosts. These included the so-called ARMAN archaea first identified in acid mine drainage (Comolli et al., 2009) and the Nanohaloarchaeota in hypersaline environments (Narasingarao et al., 2012). Initially thought to be divergent members of the Euryarchaeota, Nanoarchaeota (encompassing Nanoarchaeum), ARMAN archaea and Nanohaloarchaeota were later included in a large clade of generally fast-evolving archaea, the DPANN, that seemed to branch at the base of the archaeal tree (Rinke et al., 2013). However, the monophyly and the basal placement of some DPANN lineages remain debated, notably of Nanoarchaeota and Nanohaloarchaeota, potentially related to Thermococcales and haloarchaea or 
other Euryarchaeota, respectively (Brochier et al., 2005; Petitjean et al., 2014; Williams et al., 2017; Aouad et al., 2018). DPANN refers to the first named lineages of this group (Diapherotrites, Parvarchaeota, Aenigmarchaeota, Nanoarchaeota and Nanohaloarchaeota) but it now encompasses over ten phylum-level clades (reviewed in (Castelle and Banfield, 2018; Castelle et al., 2018; Dombrowski et al., 2019)), including Diapherotrites, Altiarchaeota and Micrarchaeota, which cluster together, and Nanoarchaeota, Parvarchaeota, Pacearchaeota, Woesearchaeota, Huberarchaeota, Nanohaloarchaeota, Aenigmarchaeota, and the recently identified Undinarchaeota (Dombrowski et al., 2020). The vast majority of these organisms were identified from single-cell genomes (SAGs) or metagenome-assembled genomes (MAGs) and remain uncultured. The analyses of these genomes show reduced metabolic capabilities suggestive of parasitic lifestyles (Dombrowski et al., 2019). Indeed, ARMAN archaea (now within Micrarchaeota) were shown to depend on Thermoplasmatales hosts in situ and in culture (Golyshina et al., 2017). However, the recent assembly of MAGs from non-acidophilic Micrarchaeota indicates wider metabolic repertoires in this group that might potentially allow for a free-living style (Kadnikov et al., 2020). Also, like in the case of Nanoarchaeum, dependency might not always imply parasitism but subtler interactions. For instance, the recently identified symbiosis of Candidatus Nanohalobium constans and its chitinolytic haloarchaeal host Halomicrobium sp. LC1Hm (Figure 2) seems to be mutualistic, at least under some conditions, and based on different polysaccharide-metabolizing activities (La Cono et al., 2020). The haloarchaeon usually degrades chitin extracellularly, producing beta-glucans that it consumes. However, in the absence of chitin, Ca. Nanohalobium ferments glycogen or starch (alpha-glucans that the host does not metabolize), making their products accessible to the host which, in turn, sustains its metabolically-limited epibiont. Nonetheless, whether this strategy maximizes the long-term fitness of the host (La Cono et al., 2020) or whether $\mathrm{Ca}$. Nanohalobium becomes parasitic in the presence of chitin and/or in the absence of glycogen or starch in the environment, remains to be established.

Similarly to the highly diverse DPANN archaea, the Candidate Phyla Radiation (CPR) was recognized a few years ago as a large clade of lineages exhibiting tiny cell size and representing a considerable proportion of bacterial diversity, essentially uncultured (Brown et al., 2015). Some of these lineages were previously known from environmental 16S rRNA gene surveys in a wide variety of ecosystems, from extreme environments to oxygen-depleted settings, including sediments and microbiomes, but others were only identified via MAGs, as their divergent rRNA sequences were missed by PCR approaches. Their genomes are also reduced to various degrees and lack genes for several essential biosynthetic capabilities, such that a symbiotic lifestyle has been proposed for this monophyletic radiation (Castelle and Banfield, 2018; Castelle et al., 2018). With the exception of one member of the Parcubacteria (OD1), identified within cells of the ciliate Paramecium bursaria (Gong et al., 2014), 
observed CPR are epibionts of prokaryotic cells, and the few described CPR members, clearly parasitic. This is the case of Nanosynbacter lyticus TM7x, a member of the CPR phylum Saccharibacteria (former TM7), which parasitizes the oral bacterium Actinomyces odontolyticus strain XH001, rapidly coevolving with its host (Bor et al., 2018) (Figure 2). Another clear example of parasitic interaction is that of Vampirococcus lugosii, a member of the CPR phylum Absconditabacteria, which preys on anoxygenic photosynthetic gammaproteobacteria of the genus Halochromatium, consuming its cytoplasmic content and killing it (Moreira et al., 2020). This is indeed the first described species of Vampirococcus, a genus of predatory bacteria first portrayed by optical and electron microscopy observations more than twenty years ago (Guerrero et al., 1986). Like TM7x, Vampirococcus has a small genome exhibiting limited metabolic capabilities. However, like some Nanohaloarchaeota (Hamm et al., 2019), it encodes for giant surface proteins likely involved in cell adhesion. The peculiar nature of CPR cell surface may be related to host recognition and infectivity (Luef et al., 2015). It is known that TM7x displays a narrow host range, being able to infect and form stable long-term relationships with specific Actinomyces species (Bor et al., 2018). This suggests a continuous arms-race of host-parasite pairs, very much as it happens in viruses and other parasites, which would sustain bilateral specificity and might explain the extraordinary diversity that is seen in some CPR and DPANN clades in environmental studies (e.g. (Belilla et al., 2019)). Actually, active mechanisms of diversification, such as diversity-generating retroelements, occur in CPR and DPANN populations, notably targeting proteins involved in attachment, defense and regulation (Paul et al., 2017).

\section{The crystal ball on prokaryotic symbioses}

In this context, we advance that research on tight physical symbiotic interactions between prokaryotes will prominently develop in the next years, revealing not only the daunting extent of their diversity but also providing unforeseen insights on their crucial ecological and evolutionary impact.

Research on CPR and DPANN phyla will continue to uncover new associated lineages (some of which have already been detected but remain unnamed) and, within recognized lineages, an increasing breadth of diversity. The generation of MAGs and SAGs will contribute to describe that diversity and reveal the metabolic potential of those lineages, the vast majority of which will be reduced, indicating a likely dependence on their hosts. This dependence will be partly corroborated if these cells turn out to be very small and physically attached to larger host cells, as has been already observed in several cases. To demonstrate the occurrence of tight physical connections, high-resolution microscopies will be useful, including scanning and electron microscopy (SEM, TEM), but also fluorescence or confocal laser scanning microscopy (CSLM) on fluorescently-labeled cells/consortia. Cryomicroscopy (cryo-TEM) 
will help to further characterize the nature of physical intercellular connections. Co-occurrence studies on natural communities combined with specific fluorescent cell-labeling techniques, including the use of antibodies (Cross et al., 2019), will help to determine specific pairs of interacting cells. This, ultimately combined with sequencing of amplified mini-metagenomes from individual consortia isolated by flowcytometry cell-sorting approaches or even manual micromanipulation, should lead to the specific identification of symbiont pairs and their eventual metabolic complementarity. Other methods, including high-resolution spectroscopic techniques on consortia incubated in enrichments or natural communities with stable isotope-labeled compounds will help to characterize the nature of transferred substrates between partners. In addition to the identification of symbiont pairs and the basis of their interaction, we expect to learn more about the biology of these organisms, including their life/infection cycle and their mode of interaction with hosts. Genome analysis of CPR and DPANN MAGs suggest that, while being strongly dependent on their host and lacking many essential biosynthetic pathways, these organisms have a more or less large repertoire of specific genes that might be involved in cell-cell adhesion, infectivity or new functions that remain to be characterized.

Beyond getting information about the biology of CPR bacteria, DPANN archaea and, potentially, other bacterial or archaeal lineages that establish tight physical intercellular connections with other bacteria and/or archaea, we will also learn about the ecological importance of these interactions. We predict that many, if not most, of the CPR bacteria and DPANN archaea play a crucial role in regulating their host population size, notably via parasitic or predatory interactions. Although a few of these lineages might have the potential for free-living style, those that are stably physically bound to their hosts are likely to have evolved obligatory symbioses. Although some of these symbioses might be mutualistic under certain environmental conditions, e.g. to collectively exploit an otherwise inaccessible substrate, under fluctuating or adverse environmental conditions, the reduced symbiont will move towards the parasitism side on the cooperation versus conflict line in detriment of the host (Figure 1). In many cases, notably of highly reduced CPR, the interactions might be essentially parasitic or predatory (leading to host cell-killing). In this sense, many CPR and DPANN lineages are likely to behave ecologically like viruses, being bloom terminators and controlling population sizes in the environments where they thrive. Furthermore, it might even be that in some complex, highly structured environments cellular parasites excel viruses in regulating prokaryotic demography. Future studies should help testing these hypotheses.

Finally, we expect that the study of these bound prokaryote-prokaryote interactions unveil important evolutionary consequences. At short evolutionary scales, we anticipate that the radiation patterns observed at the tips of DPANN and CPR phyla (and even deeper radiations) largely correlate with similar patterns in the corresponding hosts. This may be evidence for an arms race situation that 
will result in an ever-expanding diversification. This Red Queen-type of evolution is likely to be accompanied by an acceleration of evolutionary rate, especially if genome reduction implies the impairment of DNA repair pathways. Indeed, the DPANN lineages are particularly affected by fast evolving rates. Fast evolving rates but also convergent evolution (e.g. the independent reduction to parasitic lifestyles in diverse lineages), may entail problems of phylogenetic reconstruction, such as long-branch attraction artefacts. Although CPR bacteria seem less fast-evolving and truly monophyletic, controversies surrounding the phylogenetic status of DPANN archaea arise in this context. Improvement of sequence evolution models and phylogenetic methods combined with more comprehensive parasitic and non-parasitic lineages should help reconstructing the evolutionary history of these clades. Lastly, while so far many of the revealed prokaryote-prokaryote physical connections occur within the same domain, cross-domain obligatory interactions may also exist, especially since they are already known to occur facultatively (many syntrophies). Likewise, while observed intercellular interactions have been so far observed epibiotically, the possibility that endosymbioses of prokaryotes within other prokaryotes are unambiguously identified is considerably increasing. Many of these cells are very small such that endosymbioses may have been overlooked in the past, despite some sparse microscopy observations (e.g. (Wujek, 1979; Larkin et al., 1990). Furthermore, mechanisms of cell entry are known. These include not only penetration of some predatory bacteria into prey cells, such as Bdellovibrio (Rendulic et al., 2004), but, most importantly, true phagocytosis in prokaryotes, as was recently evidenced in some planctomycetes (Shiratori et al., 2019). Putative prokaryote-prokaryote endosymbioses awaiting to be discovered might, like observed ectosymbioses, move along a mutualistcompetition gradient under the regulatory effect of the environment. It is among that type of symbioses, in a particular ecological context two billion years ago, that the symbiotic consortium at the origin of the eukaryotic cell likely evolved (detailed proposed symbiogenetic models comparatively reviewed in (Spang et al., 2019; López-García and Moreira, 2020b). The identification and study of those symbioses today, if they exist, will shed precious light on eukaryogenesis.

\section{Acknowledgments}

We acknowledge support from the Moore-Simons Project on the Origin of the Eukaryotic Cell through Grant GBMF9739 (P.L.-G.) and the H2020 European Research Council Advanced Grant No. 787904 (D.M.). 


\section{References}

Aouad, M., Taib, N., Oudart, A., Lecocq, M., Gouy, M., and Brochier-Armanet, C. (2018) Extreme halophilic archaea derive from two distinct methanogen Class II lineages. Mol Phylogenet Evol 127: 4654.

Belilla, J., Moreira, D., Jardillier, L., Reboul, G., Benzerara, K., López-García, J.M. et al. (2019) Hyperdiverse archaea near life limits at the polyextreme geothermal Dallol area. Nature Ecol Evol 3: 1552-1561.

Bor, B., McLean, J.S., Foster, K.R., Cen, L., To, T.T., Serrato-Guillen, A. et al. (2018) Rapid evolution of decreased host susceptibility drives a stable relationship between ultrasmall parasite TM7X and its bacterial host. Proc Natl Acad Sci U S A 115: 12277-12282.

Brochier, C., Gribaldo, S., Zivanovic, Y., Confalonieri, F., and Forterre, P. (2005) Nanoarchaea: representatives of a novel archaeal phylum or a fast-evolving euryarchaeal lineage related to Thermococcales? Genome Biol 6: R42.

Brown, C.T., Hug, L.A., Thomas, B.C., Sharon, I., Castelle, C.J., Singh, A. et al. (2015) Unusual biology across a group comprising more than 15\% of domain Bacteria. Nature 523: 208-211.

Castelle, C.J., and Banfield, J.F. (2018) Major new microbial groups expand diversity and alter our understanding of the Tree of Life. Cell 172: 1181-1197.

Castelle, C.J., Brown, C.T., Anantharaman, K., Probst, A.J., Huang, R.H., and Banfield, J.F. (2018) Biosynthetic capacity, metabolic variety and unusual biology in the CPR and DPANN radiations. Nat Rev Microbiol 16: 629-645.

Celiker, H., and Gore, J. (2013) Cellular cooperation: insights from microbes. Trends Cell Biol 23: 9-15.

Comolli, L.R., Baker, B.J., Downing, K.H., Siegerist, C.E., and Banfield, J.F. (2009) Three-dimensional analysis of the structure and ecology of a novel, ultra-small archaeon. The ISME Journal 3: 159-167.

Cordaux, R., Bouchon, D., and Greve, P. (2011) The impact of endosymbionts on the evolution of host sex-determination mechanisms. Trends Genet 27: 332-341.

Cross, K.L., Campbell, J.H., Balachandran, M., Campbell, A.G., Cooper, S.J., Griffen, A. et al. (2019) Targeted isolation and cultivation of uncultivated bacteria by reverse genomics. Nature Biotechnology 37: 1314-1321.

Delaux, P.M., Sejalon-Delmas, N., Becard, G., and Ane, J.M. (2013) Evolution of the plant-microbe symbiotic 'toolkit'. Trends Plant Sci 18: 298-304.

Dombrowski, N., Lee, J.H., Williams, T.A., Offre, P., and Spang, A. (2019) Genomic diversity, lifestyles and evolutionary origins of DPANN archaea. FEMS Microbiol Lett 366: fnz008.

Dombrowski, N., Williams, T.A., Sun, J., Woodcroft, B.J., Lee, J.H., Minh, B.Q. et al. (2020) Undinarchaeota illuminate DPANN phylogeny and the impact of gene transfer on archaeal evolution. Nat Commun 11: 3939.

Dubilier, N., Bergin, C., and Lott, C. (2008) Symbiotic diversity in marine animals: the art of harnessing chemosynthesis. Nat Rev Microbiol 6: 725-740.

Dyall, S.D., Brown, M.T., and Johnson, P.J. (2004) Ancient invasions: from endosymbionts to organelles. Science 304: 253-257.

Fenchel, T., and Finlay, B.J. (1995) Ecology and evolution in anoxic worlds. Oxford: Oxford University Press.

Golyshina, O.V., Toshchakov, S.V., Makarova, K.S., Gavrilov, S.N., Korzhenkov, A.A., La Cono, V. et al. (2017) 'ARMAN' archaea depend on association with euryarchaeal host in culture and in situ. Nat Commun 8: 60.

Gong, J., Qing, Y., Guo, X., and Warren, A. (2014) "Candidatus Sonnebornia yantaiensis", a member of candidate division OD1, as intracellular bacteria of the ciliated protist Paramecium bursaria (Ciliophora, Oligohymenophorea). Systematic and Applied Microbiology 37: 35-41. 
Gorter, F.A., Manhart, M., and Ackermann, M. (2020) Understanding the evolution of interspecies interactions in microbial communities. Philos Trans R Soc Lond B Biol Sci 375: 20190256.

Gourion, B., Berrabah, F., Ratet, P., and Stacey, G. (2015) Rhizobium-legume symbioses: the crucial role of plant immunity. Trends Plant Sci 20: 186-194.

Guerrero, R., Pedros-Alio, C., Esteve, I., Mas, J., Chase, D., and Margulis, L. (1986) Predatory prokaryotes: predation and primary consumption evolved in bacteria. Proc Natl Acad Sci U S A 83: 21382142.

Hamm, J.N., Erdmann, S., Eloe-Fadrosh, E.A., Angeloni, A., Zhong, L., Brownlee, C. et al. (2019) Unexpected host dependency of Antarctic Nanohaloarchaeota. Proc Natl Acad Sci U S A 116: 1466114670.

Hosokawa, T., Koga, R., Kikuchi, Y., Meng, X.Y., and Fukatsu, T. (2010) Wolbachia as a bacteriocyteassociated nutritional mutualist. Proc Natl Acad Sci U S A 107: 769-774.

Huber, H., Hohn, M.J., Rachel, R., Fuchs, T., Wimmer, V.C., and Stetter, K.O. (2002) A new phylum of Archaea represented by a nanosized hyperthermophilic symbiont. Nature 417: 63-67.

Husnik, F., and Keeling, P.J. (2019) The fate of obligate endosymbionts: reduction, integration, or extinction. Curr Opin Genet Dev 58-59: 1-8.

Imachi, H., Nobu, M.K., Nakahara, N., Morono, Y., Ogawara, M., Takaki, Y. et al. (2020) Isolation of an archaeon at the prokaryote-eukaryote interface. Nature 577: 519-525.

Jahn, U., Gallenberger, M., Paper, W., Junglas, B., Eisenreich, W., Stetter, K.O. et al. (2008) Nanoarchaeum equitans and Ignicoccus hospitalis: new insights into a unique, intimate association of two archaea. J Bacteriol 190: 1743-1750.

Kadnikov, V.V., Savvichev, A.S., Mardanov, A.V., Beletsky, A.V., Chupakov, A.V., Kokryatskaya, N.M. et al. (2020) Metabolic diversity and evolutionary history of the archaeal Phylum "Candidatus Micrarchaeota" uncovered from a freshwater lake metagenome. Appl Environ Microbiol 86: e0219902120.

Keeling, P.J. (2013) The number, speed, and impact of plastid endosymbioses in eukaryotic evolution. Annu Rev Plant Biol 64: 583-607.

La Cono, V., Messina, E., Rohde, M., Arcadi, E., Ciordia, S., Crisafi, F. et al. (2020) Symbiosis between nanohaloarchaeon and haloarchaeon is based on utilization of different polysaccharides. Proc Natl Acad Sci U S A online early: 202007232.

Larkin, J.M., Henk, M.C., and Burton, S.D. (1990) Occurrence of a Thiothrix sp. attached to mayfly larvae and presence of parasitic bacteria in the Thiothrix sp. Appl Environ Microbiol 56: 357-361.

Leclercq, S., Theze, J., Chebbi, M.A., Giraud, I., Moumen, B., Ernenwein, L. et al. (2016) Birth of a W sex chromosome by horizontal transfer of Wolbachia bacterial symbiont genome. Proc Natl Acad Sci U S A: pii: 201608979. [Epub ahead of print].

Little, A.E.F., Robinson, C.J., Peterson, S.B., Raffa, K.F., and Handelsman, J. (2008) Rules of Engagement: Interspecies Interactions that Regulate Microbial Communities. Annual Review of Microbiology 62: 375401.

Lopez-Garcia, P., Eme, L., and Moreira, D. (2017) Symbiosis in eukaryotic evolution. J Theor Biol 434: 20-33.

López-García, P., and Moreira, D. (2020a) Cultured Asgard archaea shed light on eukaryogenesis. Cell 181: 232-235.

López-García, P., and Moreira, D. (2020b) The Syntrophy hypothesis for the origin of eukaryotes revisited. Nat Microbiol 5: 655-667.

Lovley, D.R. (2017) Syntrophy Goes Electric: Direct Interspecies Electron Transfer. Annu Rev Microbiol 71: 643-664.

Luef, B., Frischkorn, K.R., Wrighton, K.C., Holman, H.Y., Birarda, G., Thomas, B.C. et al. (2015) Diverse uncultivated ultra-small bacterial cells in groundwater. Nat Commun 6: 6372.

McCutcheon, J.P., and Moran, N.A. (2012) Extreme genome reduction in symbiotic bacteria. Nat Rev Microbiol 10: 13-26. 
Mitri, S., and Foster, K.R. (2013) The genotypic view of social interactions in microbial communities. Annu Rev Genet 47: 247-273.

Monteil, C.L., Vallenet, D., Menguy, N., Benzerara, K., Barbe, V., Fouteau, S. et al. (2019) Ectosymbiotic bacteria at the origin of magnetoreception in a marine protist. Nat Microbiol 4: 1088-1095.

Moran, N.A., and Bennett, G.M. (2014) The tiniest tiny genomes. Annu Rev Microbiol 68: 195-215.

Moreira, D., Zivanovic, Y., Lopez-Archilla, A.I., Iniesto, M., and Lopez-Garcia, P. (2020) Reductive evolution and unique infection and feeding mode in the CPR predatory bacterium Vampirococcus lugosii. bioRxiv: 2020.2011.2010.374967.

Morris, B.E., Henneberger, R., Huber, H., and Moissl-Eichinger, C. (2013) Microbial syntrophy: interaction for the common good. FEMS Microbiol Rev 37: 384-406.

Moscoviz, R., Quéméner, E.D., Trably, E., Bernet, N., and Hamelin, J. (2020) Novel Outlook in Microbial Ecology: Nonmutualistic Interspecies Electron Transfer. Trends Microbiol 28: 245-253.

Moya, A., Pereto, J., Gil, R., and Latorre, A. (2008) Learning how to live together: genomic insights into prokaryote-animal symbioses. Nat Rev Genet 9: 218-229.

Nadell, C.D., Drescher, K., and Foster, K.R. (2016) Spatial structure, cooperation and competition in biofilms. Nat Rev Microbiol 14: 589-600.

Narasingarao, P., Podell, S., Ugalde, J.A., Brochier-Armanet, C., Emerson, J.B., Brocks, J.J. et al. (2012) De novo metagenomic assembly reveals abundant novel major lineage of Archaea in hypersaline microbial communities. ISME J 6: 81-93.

Nowack, E.C., and Melkonian, M. (2010) Endosymbiotic associations within protists. Philos Trans R Soc Lond B Biol Sci 365: 699-712.

Obeng, N., Pratama, A.A., and Elsas, J.D.V. (2016) The Significance of Mutualistic Phages for Bacterial Ecology and Evolution. Trends Microbiol 24: 440-449.

Overmann, J., and Van Gemerden, H. (2000) Microbial interactions involving sulfur bacteria: implications for the ecology and evolution of bacterial communities. FEMS Microbiol Ecol 24: 591-599. Paul, B.G., Burstein, D., Castelle, C.J., Handa, S., Arambula, D., Czornyj, E. et al. (2017) Retroelementguided protein diversification abounds in vast lineages of Bacteria and Archaea. Nat Microbiol 2: 17045. Petitjean, C., Deschamps, P., Lopez-Garcia, P., and Moreira, D. (2014) Rooting the domain archaea by phylogenomic analysis supports the foundation of the new kingdom proteoarchaeota. Genome Biol Evol 7: 191-204.

Podar, M., Anderson, I., Makarova, K.S., Elkins, J.G., Ivanova, N., Wall, M.A. et al. (2008) A genomic analysis of the archaeal system Ignicoccus hospitalis-Nanoarchaeum equitans. Genome Biol 9: R158.

Rendulic, S., Jagtap, P., Rosinus, A., Eppinger, M., Baar, C., Lanz, C. et al. (2004) A predator unmasked: life cycle of Bdellovibrio bacteriovorus from a genomic perspective. Science 303: 689-692.

Rinke, C., Schwientek, P., Sczyrba, A., Ivanova, N.N., Anderson, I.J., Cheng, J.F. et al. (2013) Insights into the phylogeny and coding potential of microbial dark matter. Nature 499: 431-437.

Roossinck, M.J. (2011) The good viruses: viral mutualistic symbioses. Nat Rev Microbiol 9: 99-108.

Roossinck, M.J., and Bazán, E.R. (2017) Symbiosis: Viruses as Intimate Partners. Annu Rev Virol 4: $123-$ 139.

Sassera, D., Beninati, T., Bandi, C., Bouman, E.A., Sacchi, L., Fabbi, M., and Lo, N. (2006) 'Candidatus Midichloria mitochondrii', an endosymbiont of the tick Ixodes ricinus with a unique intramitochondrial lifestyle. Int J Syst Evol Microbiol 56: 2535-2540.

Schwartz, R.M., and Dayhoff, M.O. (1978) Origins of prokaryotes, eukaryotes, mitochondria, and chloroplasts. Science 199: 395-403.

Shiratori, T., Suzuki, S., Kakizawa, Y., and Ishida, K.-I. (2019) Phagocytosis-like cell engulfment by a planctomycete bacterium. Nat Commun 10: 5529-5529.

Sieber, J.R., Mclnerney, M.J., and Gunsalus, R.P. (2012) Genomic insights into syntrophy: the paradigm for anaerobic metabolic cooperation. Annu Rev Microbiol 66: 429-452.

Spang, A., Stairs, C.W., Dombrowski, N., Eme, L., Lombard, J., Caceres, E.F. et al. (2019) Proposal of the reverse flow model for the origin of the eukaryotic cell based on comparative analyses of Asgard archaeal metabolism. Nat Microbiol. 
Spang, A., Saw, J.H., Jorgensen, S.L., Zaremba-Niedzwiedzka, K., Martijn, J., Lind, A.E. et al. (2015) Complex archaea that bridge the gap between prokaryotes and eukaryotes. Nature 521: 173-179.

Thompson, A.W., Foster, R.A., Krupke, A., Carter, B.J., Musat, N., Vaulot, D. et al. (2012) Unicellular cyanobacterium symbiotic with a single-celled eukaryotic alga. Science 337: 1546-1550.

von Dohlen, C.D., Kohler, S., Alsop, S.T., and McManus, W.R. (2001) Mealybug beta-proteobacterial endosymbionts contain gamma-proteobacterial symbionts. Nature 412: 433-436.

Waters, E., Hohn, M.J., Ahel, I., Graham, D.E., Adams, M.D., Barnstead, M. et al. (2003) The genome of Nanoarchaeum equitans: Insights into early archaeal evolution and derived parasitism. Proc Natl Acad Sci U S A 17: 17.

Werren, J.H., Baldo, L., and Clark, M.E. (2008) Wolbachia: master manipulators of invertebrate biology. Nat Rev Microbiol 6: 741-751.

Williams, T.A., Szöllősi, G.J., Spang, A., Foster, P.G., Heaps, S.E., Boussau, B. et al. (2017) Integrative modeling of gene and genome evolution roots the archaeal tree of life. Proc Natl Acad Sci U S A 114: E4602-e4611.

Wujek, D.E. (1979) Intracellular bacteria in the blue-green alga Pleurocapsa minor Trans Am Microscop Soc 98: 143-145

Zaremba-Niedzwiedzka, K., Caceres, E.F., Saw, J.H., Backstrom, D., Juzokaite, L., Vancaester, E. et al. (2017) Asgard archaea illuminate the origin of eukaryotic cellular complexity. Nature 541: 353-358. 


\section{Figure legends}

Figure 1. Biological interactions in microbial communities along the cooperation-competition continuum. In complex communities, microorganisms can entertain direct, facultative interactions with other community members, which can vary from cooperation (fitness increases in all interacting members) to competition (implying fitness decrease in at least some interacting members). Stable physical connection between interacting microorganisms often lead to obligatory symbioses. The nature of interactions within symbiotic consortia can also fluctuate along a mutualism (cooperative, synergistic) - parasitism (antagonistic) continuum, with fitness increasing, remaining neutral or decreasing for one or the two partners. In both, facultative interactions in complex communities or obligatory symbioses, the environmental conditions determine the nature of the interactions between microbial partners.

Figure 2. Examples of prokaryote-prokaryote obligatory symbioses. A, scanning electron microscopy (SEM) image showing Nanosynbacter lyticus TM7x, a member of the CPR phylum Saccharibacteria (former TM7), parasitizing its bacterial host Actinomyces odontolyticus strain XH001 (Bor et al., 2018) (courtesy of B. Bor). B, SEM picture of Candidatus Nanohalobium constans LC1Nh associated to its chitinolytic haloarchaeal host Halomicrobium sp. LC1Hm (La Cono et al., 2020) (courtesy M. M. Yakimov). C, transmission electron microscopy (TEM) image of Nanoarchaeum equitans associated to its hyperthermophilic archaeal host Ignicoccus hospitalis (Huber et al., 2002) (courtesy K.O. Stetter). D, TEM picture of the SR1 phylum (CPR) member Vampirococcus lugosii parasitizing its anoxygenic photosynthetic bacterial host Halochromatium sp. (Moreira et al., 2020). The bar size corresponds to $500 \mathrm{~nm}$. 


\section{The selective balance}

Ecological interactions (symbiotic or not)

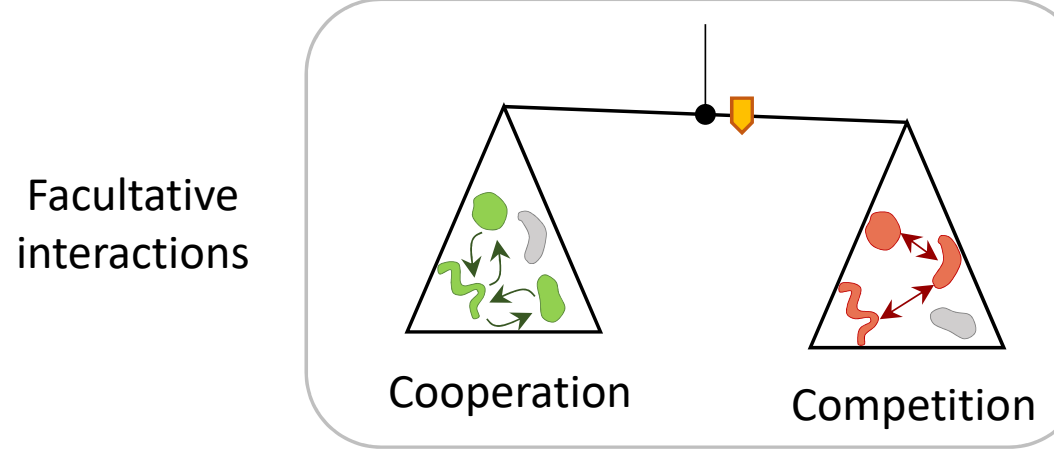

Communities

Symbiosis

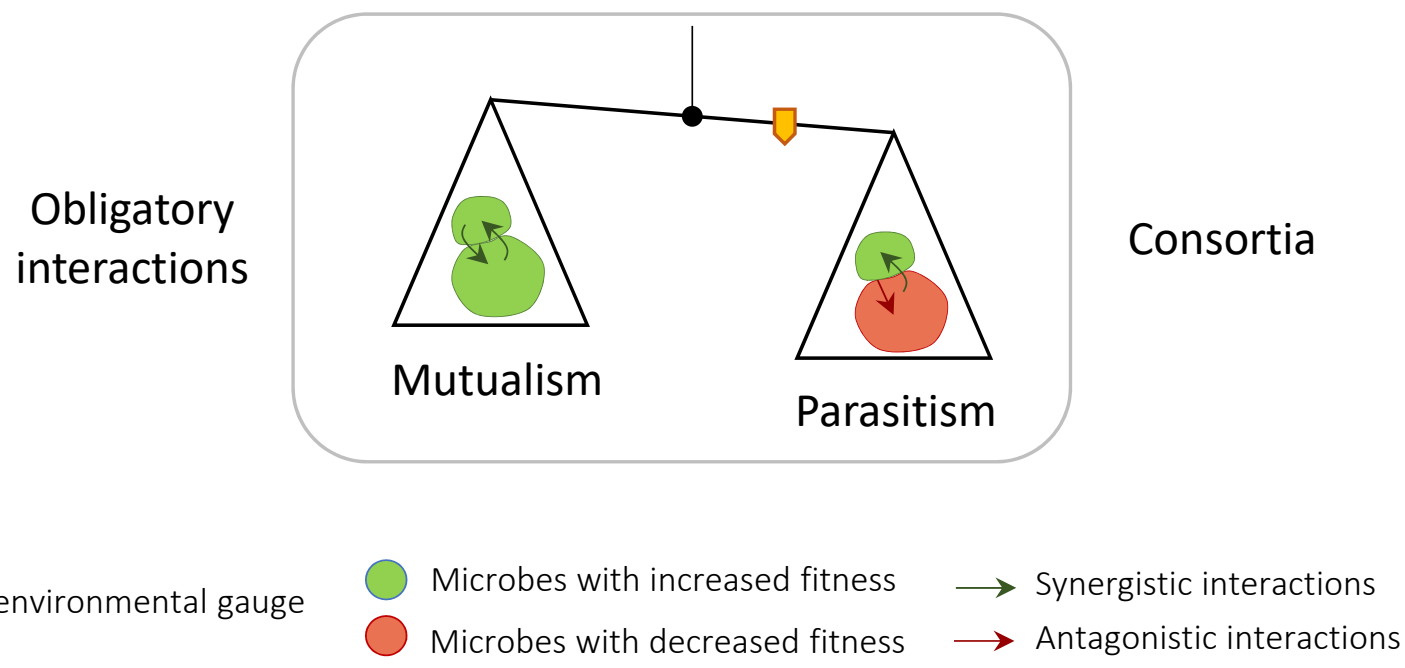

Figure 1. Lopez-Garcia \& Moreira 

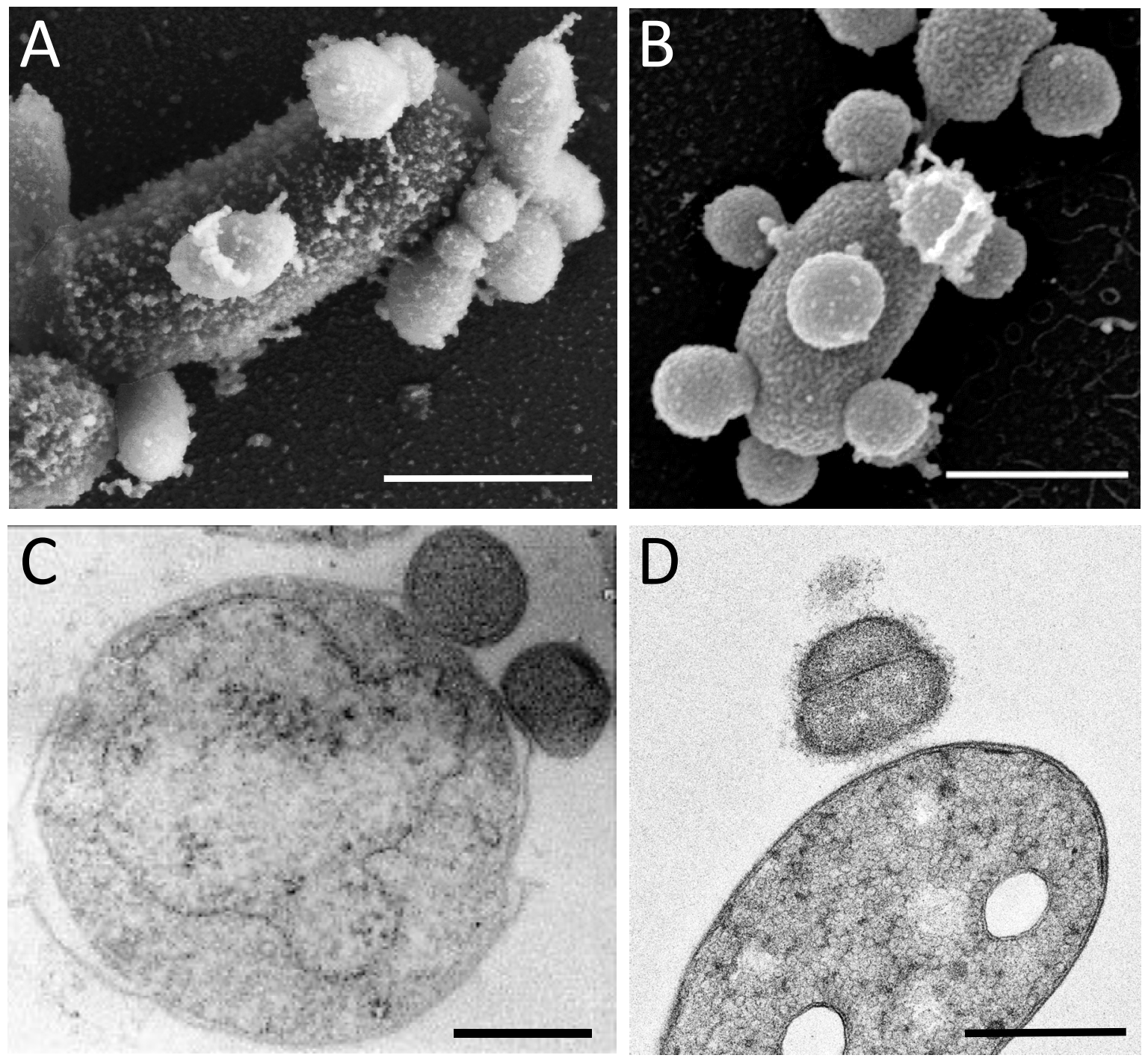

Figure 2. Lopez-Garcia \& Moreira 